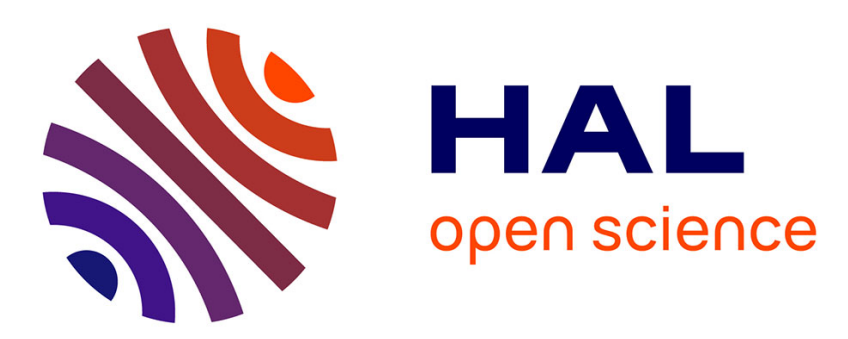

\title{
Filleting sharp edges of multi-partitioned volume finite element meshes
}

Ruding Lou, Jean-Philippe Pernot, Franca Giannnini, Philippe Veron, Bianca Falcidieno

\section{- To cite this version:}

Ruding Lou, Jean-Philippe Pernot, Franca Giannnini, Philippe Veron, Bianca Falcidieno. Filleting sharp edges of multi-partitioned volume finite element meshes. Engineering Computations, 2015, 32 (1), pp.129-154. 10.1108/EC-03-2013-0074 . hal-01118671

\section{HAL Id: hal-01118671 \\ https://hal.science/hal-01118671}

Submitted on 1 Apr 2015

HAL is a multi-disciplinary open access archive for the deposit and dissemination of scientific research documents, whether they are published or not. The documents may come from teaching and research institutions in France or abroad, or from public or private research centers.
L'archive ouverte pluridisciplinaire HAL, est destinée au dépôt et à la diffusion de documents scientifiques de niveau recherche, publiés ou non, émanant des établissements d'enseignement et de recherche français ou étrangers, des laboratoires publics ou privés. 


\title{
Filleting sharp edges of multi-partitioned volume finite element meshes
}

\author{
Ruding Lou \\ Institut Image - Le2i UMR CNRS 6306, Arts et Metiers ParisTech, \\ Chalon-sur-Saone, France \\ Jean-Philippe Pernot \\ CNRS-LSIS, Arts et Metiers ParisTech, Aix-en-Provence, France \\ Franca Giannini \\ IMATI-CNR, Consiglio Nazionale delle Ricerche, Genoa, Italy \\ Philippe Veron \\ CNRS-LSIS, Arts et Metiers ParisTech, Aix-en-Provence, France, and \\ Bianca Falcidieno \\ IMATI-CNR, Consiglio Nazionale delle Ricerche, Genoa, Italy
}

\begin{abstract}
Purpose - The purpose of this paper is to set up a new framework to enable direct modifications of volume meshes enriched with semantic information associated to multiple partitions. An instance of filleting operator is prototyped under this framework and presented in the paper.

Design/methodology/approach - In this paper, a generic mesh modification operator has been designed and a new instance of this operator for filleting finite element (FE) sharp edges of tetrahedral multi-partitioned meshes is also pro-posed. The filleting operator works in two main steps. The outer skin of the tetrahedral mesh is first deformed to round user-specified sharp edges while satisfying constraints relative to the shape of the so-called Virtual Group Boundaries. Then, in the filleting area, the positions of the inner nodes are relaxed to improve the aspect ratio of the mesh elements.

Findings - The classical mainstream methodology for product behaviour optimization involves the repetition of four steps: $\mathrm{CAD}$ modelling, meshing of $\mathrm{CAD}$ models, enrichment of models with $\mathrm{FE}$ simulation semantics and FEA. This paper highlights how this methodology could be simplified by two steps: simulation model modification and FEA. The authors set up a new framework to enable direct modifications of volume meshes enriched with semantic information associated to multiple partitions and the corresponding fillet operator is devised.

Research limitations/implications - The proposed framework shows only a paradigm of direct modifications of semantic enriched meshes. It could be further more improved by adding or changing the modules inside. The fillet operator does not take into account the exact radius imposed by user. With this proposed fillet operator the mesh element density may not be enough high to obtain wished smoothness.
\end{abstract}

Originality/value - This paper fulfils an identified industry need to speed up the product behaviour analysis process by directly modifying the simulation semantic enriched meshes.

Keywords Computer aided design, FE analysis, Fillet, Finite element mesh, Sharp edge,

Tetrahedral mesh

\section{Nomenclature}

CAD: $\quad$ Computer Aided Design

BC: $\quad$ Boundary Condition

FEA: Finite Element Analysis

VGB: Virtual Group Boundary 


\section{Introduction}

Computer-aided tools provide important improvements both in cost and performance in all the product life cycle activities, from design to manufacturing, distribution, disposal and maintenance. In the last decades they have been collected in the so-called PLM systems which additionally provide suitable means for the organization and archival of the produced data. Among them, Finite Element Analysis (FEA) plays a key role for evaluating design solutions in the context of product design and maintenance. Actually, FEA helps reducing costs while minimising the number of physical prototypes and shortening the time to market in case of new products. Actually, it reduces many costs due to the experimental validation steps of a newly designed solution. The virtual prototyping enables the assessment of various optimized solutions and reduces time-consuming and expensive physical prototyping. Therefore the process is improved not only by reducing the cost but also allowing the evaluation of more alternatives and consequently the achievement of better quality products and solutions. All these advantages are very valuable for competitive engineering and efficient industrial maintenance studies. Since FEA represents a key element for industrial companies using numerical studies, improving the FEA workflow is crucial to further reduce time and cost while preserving the quality of numerical studies.

Classically, the mainstream methodology for product behaviour analysis involves the repetition of four steps: $\mathrm{CAD}$ modelling, meshing of CAD models, enrichment of models with finite element $(\mathrm{FE})$ simulation semantics, and the $\mathrm{FE}$ analysis. The specification of simulation semantics often consists in defining the material behaviour law, the boundary conditions (BCs) in terms of loads, fixations, etc. Figure 1(a)-(d) show an example of this process illustrating these four steps for the design of a hook. The simulation semantics defined on the FE model of the hook are: steel material, fixation of the upper part and loading (Figure 1(c)). On this example, the FEA result indicates a stress concentration on the designed structure. Figure 1(e) presents the zoom of the initially designed CAD model, and the corresponding FEA result is shown in Figure 1(f). As usual, the stress

Figure 1.

Example of a hook design using the classical CAE loop (a-d)

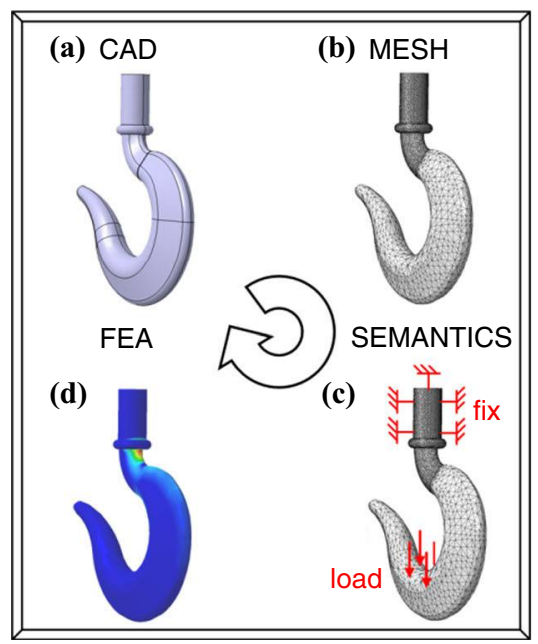

(e)

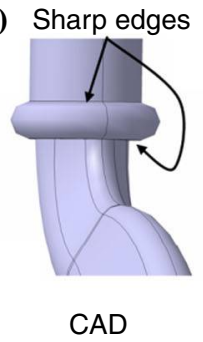

(f) Stress concentration

(g)

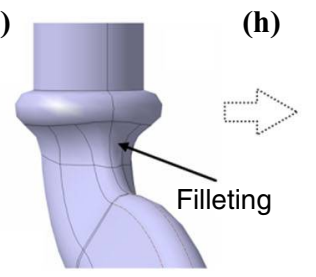

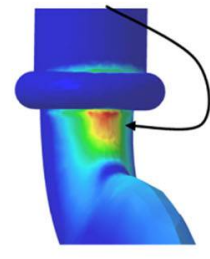

FEA

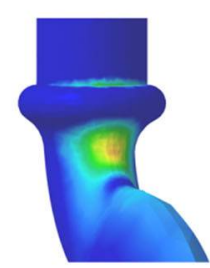

Note: Sharp edges induce a stress concentration (e), (f) that is decreased when filleting the edges $(\mathrm{g}),(\mathrm{h})$ 
concentrates around the sharp edges of the structure near the $\mathrm{BC}$ application zone. In the case of this specific hook, the local stress constraints can reach $153 \mathrm{MPa}$. To avoid this stress concentration problem, a solution consists in filleting the corresponding sharp edges. Therefore, the CAD model is locally modified (Figure $1(\mathrm{~g})$ ), the FE mesh is recreated and all the BCs are redefined. The new FEA result (Figure 1(h)) shows a reduction of the stress in the problematic area around 20 per cent for a given mesh finesse.

Even if such a modification process has now become very common for optimizing the shape of products, it still suffers from some drawbacks. Actually, the FE model preparation steps (steps 1-3) as well as the simulation step (step 4) are time-consuming. This is even more true when considering that the design process normally requires a succession of optimization loops where the identified four steps are repeated several times before converging to the optimal solution. Unfortunately, even a small change in the CAD model requires the updating of all the other steps with several repeated actions. These aspects are addressed in Lou et al. (2010a, b)). Recently, an alternative method to FEA has appeared (Cottrell et al., 2009). Isogeometric Analysis allows performing $\mathrm{FE}$ simulations directly on CAD-models. This innovative product design evaluation mode would not need anymore the transfer from a geometry-oriented design model to a FE-based computational model. However, in industrial practises, the CAD model is not necessarily available and it is mandatory to develop a new approach of doing the modifications directly on FE models without going back to the CAD models. Thanks to the last it is possible to generate and to evaluate alternative solutions directly on enriched meshes.

Therefore, to reduce the time of the numerical study and to avoid going back to the $\mathrm{CAD}$ models during the optimization design process, we have been working on the definition of a general CAD-less fast prototyping approach (Lou et al., 2010b). In this paper, the various modules of our CAD-less approach are restructured to better highlight the "generic" character of the proposed CAD-less operator working directly on enriched FE meshes. A newly developed instance of the generalized operator is also presented here. It concerns a filleting operator directly rounding $2 \mathrm{D}$ (triangular) and 3D (tetrahedral) enriched FE meshes along user-specified sharp edges. The proposed operator is based on a local mesh deformation technique detailed in the paper. This approach can be integrated in advanced systems used within a PLM environment, where the versions of the considered alternative solutions, possibly sharing the same set of FEA semantic information, can be stored. Our current focus is on the direct modification of the FEA model, and not on the management aspect of the exploited and obtained data resulting from the modification.

Our paper is organized as follows. Related works are analysed in Section 2, according to some pertinent criteria introduced in Section 2.1. The complete architecture of the CAD-less operator framework is presented (Section 3). Then, the mesh filleting operator, i.e. an instance of our generic operator, is detailed (Section 4). Finally, the application of the filleting operator on both triangle and tetrahedral meshes is illustrated (Section 5). At the end, the conclusion and future works are discussed.

\section{State-of-the-art}

\subsection{Hypotheses and restrictions}

As stated in the introduction, this paper neither addresses the way FE semantics (BCs, material behaviour laws, etc.) can be associated to geometric entities, nor how those crucial information can be updated and exchanged during the simulation model preparation steps. In spite of this, we assume that the semantic information has been 
attached to the FE meshes through the use of multiple partitions, and we elaborate on the way those enriched meshes can be directly shaped and filleted without going back to the $\mathrm{CAD}$ model. In this sense, all the discussions and references to the way such a process could be set up in a more interoperable manner are skipped since it would require a standalone paper to present and analyse all the existing and on-going approaches. In addition, we do not address the way the initial mesh is obtained and potentially refined. Moreover, we do not address isogeometric analysis nor mesh-less approaches discussed in the introduction. Instead, we focus more on the geometric approaches as well as on the way these methods can work on already existing semantically enriched meshes

\subsection{Adopted analysis criteria for the analysis of mesh operator for FE simulation}

Clearly, to be really efficient and avoid multiple and time-consuming iterative updates of CAD models as well as tedious re-meshing steps of potentially complex parts and products, such a CAD-less framework cannot only consider the geometric aspects. Also the treatment of the semantics, associated to the geometric entities through the use of partitions and groups, has to be considered. Actually, the link between the geometric entities and the semantic information often results from the specification of an intermediate layer made of groups. FE groups can contain nodes, edges, faces and 3D elements (e.g. tetrahedrons, hexahedra) as well as a mix of them. To perform an accurate state-of-the-art survey in this domain, two categories of criteria have been identified and detailed hereunder. Each approach is then analysed according to those criteria and a symbol "+" (resp. "-") is used when the approach meet (resp. does not meet) a criterion.

Considering the geometric aspects of the direct mesh modification operators, five criteria can be used to analyse the proposed approaches:

(1) Local vs global modifications: this criterion is used to discriminate approaches that would modify locally the mesh from those modifying it globally. For example, a method that would re-mesh entirely the inner part of a volume mesh does not meet this criterion $\left(\mathrm{a}^{-}\right)$, whereas an approach whose modification can be restricted to a subpart of the mesh will meet this criterion $\left(\mathrm{a}^{+}\right)$. Actually, enriched FE meshes have often been tuned and validated with respect to experimental results. It is therefore crucial to limit as much as possible the extent of the changes so that the modelling hypotheses and local adaptations remain valid.

(2) Preservation of the initial shape: even if the operator affects a subpart of the mesh $\left(\mathrm{a}^{+}\right)$, it is important to do so that the type of the external skin of the mesh is not affected and remains the same. For example, the insertion of a through hole inside a cube-like tetrahedral mesh should keep the six outer faces planar $\left(\mathrm{b}^{+}\right)$without introducing any displacements of the outer triangles out of the six planes $\left(\mathrm{b}^{-}\right)$.

(3) Aspect ratio of the mesh elements: this criterion is used to qualify the modifications with respect to the evolution of the aspect ratio of the mesh elements in the modified area. The aspect ratio measures the deviation between a mesh element and an ideal one having all its edges of equal length. When the aspect ratio is equal to 1 , the element is considered as ideal. When the aspect ratio is equal to 0 , it means that the element is flat and has no volume and no area. Thus, long, thin and skinny elements have a low aspect ratio (Ciarlet, 1978). The aspect ratios of the modified elements can remain the same or can be 
even better $\left(\mathrm{c}^{+}\right)$, or they can be worst $\left(\mathrm{c}^{-}\right)$than the elements of the original mesh. It is commonly admitted that an element is a good one, with respect to the FE approximation, if the aspect ratio of the worst element is greater than 0.5.

(4) Self-intersecting elements: this criterion is used to qualify the capability to avoid self-intersections when performing mesh modifications $\left(\mathrm{d}^{+}\right)$. The generation of self-intersections would lead to undesirable simulation results $\left(\mathrm{d}^{-}\right)$.

(5) Modification tool shapes: this criterion indicates whether the modified areas follow the shapes of the modification tool $\left(\mathrm{e}^{+}\right)$or not $\left(\mathrm{e}^{-}\right)$. For example, when drilling a tetrahedral mesh with a cylindrical surface, there should be a part of the external skin of the modified mesh that should approximate the cylindrical tool. When talking about a filleting operator, this criterion is also relative to the capacity to handle sharp edges in different configurations. Actually, there are two different types of corner: interior (concave) and exterior (convex). When the dihedral angle between two triangles sharing a sharp edge is smaller than $180^{\circ}$, it is an interior corner configuration otherwise it is an exterior corner configuration. Examples can be seen in the Figure 2. The topology of the sharp edges could be differentiated into three sets: cycled (Figure 2(a)), with termination (Figure 2(c)) and branched (Figure 2(e)). Considering the FE semantics required for the simulation and associated to the geometric elements through the use of groups, two criteria can be used to analyse the various approaches.

(6) Treatment of mesh entity groups: this criterion checks whether the group definition of the modified mesh elements is maintained $\left(\mathrm{f}^{+}\right)$or not $\left(\mathrm{f}^{-}\right)$. Actually, the group definition has to be preserved in the unmodified areas, whereas the group definition has to be updated in the modified zone. This criterion is also used to distinguish the methods that would not use the groups' definition as constraints for the geometric modifications $\left(\mathrm{f}^{-}\right)$from those which would use them $\left(\mathrm{f}^{+}\right)$. In some sense, it helps evaluating how much an approach works on enriched FE meshes $\left(\mathrm{f}^{+}\right)$or simply on semantic-free meshes $\left(\mathrm{f}^{-}\right)$.

(7) Treatment of physical semantics: this criterion indicates whether the semantic information associated with the different groups are preserved $\left(\mathrm{g}^{+}\right)$or not $\left(\mathrm{g}^{-}\right)$. This evaluation also takes into account the way an update of a group involves an update of the associated physical semantics. The update of physical semantics (e.g. external loads or fixing conditions) can either be automatic or semi-automatic, and strongly depends on the nature of the semantic information. For example, a fluid may impose a pressure on the internal faces of a caisson, and this semantic information might therefore be propagated when modifying the inner shape of the caisson. This refers to the notions of inheritance and propagation mechanisms that have to be incorporated into the definition of those high level operators $\left(\mathrm{g}^{+}\right)$. Of course, the expert still controls the process and has to assist and validate the proposed treatments.

\subsection{Related works}

In the literature, many works address the way meshes can be modified directly, but few of them fulfill the above mentioned criteria. The Boolean operations proposed by Krsek (Premysl, 2002) are performed on a volume mesh by doing intersection of boundary meshes and completely refilling the tetrahedral mesh. The full re-meshing produces good quality of mesh elements $\left(\mathrm{c}^{+}\right)$but this is not admissible when manipulating tuned 

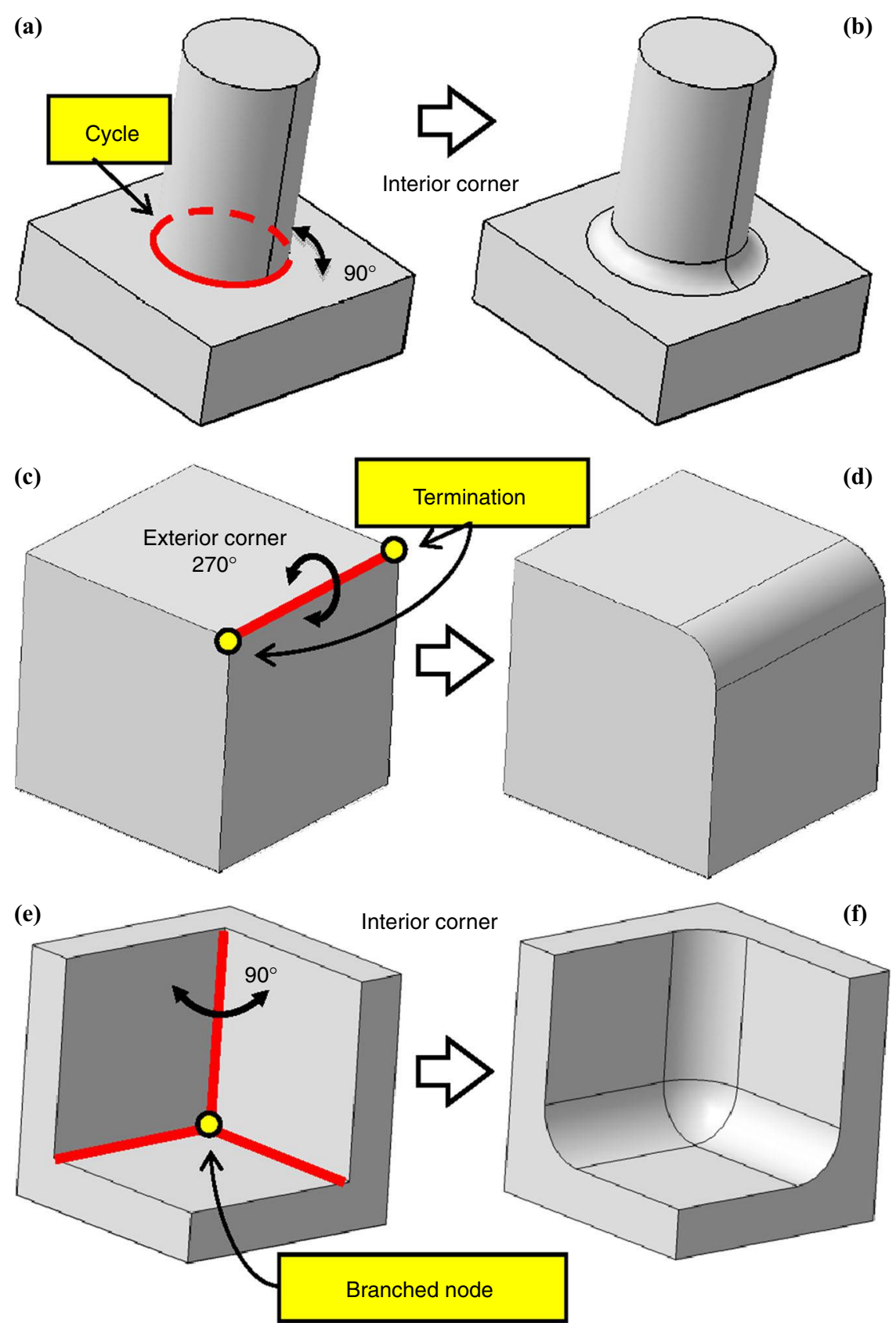

Figure 2.

Examples of exterior corner (c) and interior corner (a and c).

Sharp edge in a ring (a), sharp edge with terminations (c) and multiple sharp edge branches (b) 
FE meshes for which only local modifications are allowed $\left(\mathrm{a}^{-}\right)$. Similarly, an approach to insert crack feature into a volume mesh has been designed in Bremberg and Dhondt (2008). The intersection between the crack tool surface mesh and the boundary mesh of the volume mesh is computed and a complete re-meshing is performed. The quality of the mesh elements and the shape of the modification tool are checked $\left(\mathrm{c}^{+}, \mathrm{e}^{+}\right)$but the modifications are not local $\left(\mathrm{a}^{-}\right)$.

The Boolean operations performed on triangle meshes (Biermann et al., 2001) uses the Loop's subdivision technique (Loop, 1987) in order to approximate the intersection. The shape of the intersection zone is not exact $\left(\mathrm{e}^{-}\right)$. The intersection repairing in case of mesh offsetting (Jung et al., 2004) and the mesh cutting approach proposed in Dakowicz and Gold (2005) ensure local modifications on mesh $\left(\mathrm{a}^{+}\right)$ but do not satisfy the criterion relative to the quality of the mesh elements $\left(\mathrm{c}^{-}\right)$. Turini et al. (2006) have proposed cutting mesh approach that directly subdivides and removes mesh elements in contact with the tool, in order to approximate the cutting path Turini et al., 2006. The quality of the mesh elements is not ensured $\left(\mathrm{c}^{-}\right)$and the shape of the modification tool is not respected $\left(\mathrm{e}^{-}\right)$. Chouadria et al. (2006) have proposed an approach for the treatment of digital mock-up assemblies (Chouadria and Veron, 2006). The modifications are local $\left(\mathrm{a}^{+}\right)$but self-intersecting elements are not avoided $\left(d^{-}\right)$. The operator proposed by Chen (2007) overcomes difficulties in many degenerated cases such as the two input models being in contact only on an edge. The conversion from surface model to volumetric is necessary and the quality of the produced triangles is not addressed $\left(\mathrm{c}^{-}\right)$.

Concerning the filleting of sharp edges, several approaches refer to as mesh offsetting and mesh filleting. Generally speaking, the mesh offsetting technique cannot ensure local modifications $\left(\mathrm{a}^{-}\right)$and it cannot be applicable when there are interior and exterior corners on a model $\left(\mathrm{e}^{-}\right)$. A surface mesh offsetting approach for scaling up the model is presented in Kim et al. (2004). It allows producing rounded feature on a convex sharp corner. The presented method does not take care of the quality of the mesh elements $\left(c^{-}\right)$and it does not avoid self-intersecting elements $\left(\mathrm{d}^{-}\right)$. In addition, the offsetting is not a local modification $\left(\mathrm{a}^{-}\right)$. The operator does not cover the sharp edges that are concave because after offsetting it would produce self-intersecting configurations. Moreover, using this approach, it is difficult to treat complex sharp edge terminations $\left(\mathrm{e}^{-}\right)$, i.e. configurations where sharp edges do not form a simple loop (Figure 2(c)). The proposed mesh offset method is then extended to manufacturing operators, e.g. material cutting (Kim and Yang, 2005). The material removing from a model is realized by offsetting the surface.

Another polygonal mesh filleting operator based on offsetting is also proposed by Chen et al. (2005). They propose two steps surface offsetting: shrinking and growing. The round transition surface is generated by growing up the shrunken mesh model. Therefore the method would change locally the fillet zone $\left(\mathrm{a}^{+}\right)$. By the way, no mesh element quality optimization is performed $\left(c^{-}\right)$. In addition with their method it is difficult to fillet sharp edge terminations $\left(\mathrm{e}^{-}\right)$. Further offsetting (growing and shrinking) are proposed by Pavic and Kobbelt (2008) allowing to preserve the features of models $\left(\mathrm{b}^{+}\right)$. The uniformity of arbitrary offset distance is maintained in Chen and Wang (2011).

In Hui and Lai (2006), a subdivision technique is used for smoothing a transition zone in a blended model. For blending two meshes, the intersection mesh elements are removed and a rough transition mesh is created to connect the two models. This created mediator mesh is subdivided until the transition from one to the 
other is smooth enough. Mesh refinement plus geometric fairing algorithm is proposed in Igarashi and Hughes (2003). These techniques could be used to fillet a sharp edge on a mechanical model and the modification could be constrained in a local zone $\left(\mathrm{a}^{+}\right)$. But it is anyhow difficult to cover the case of sharp edge terminations $\left(\mathrm{e}^{-}\right)$.

Surface mesh rounding/blending operations using the rolling-ball method are defined in Lee et al. (2001), Liu et al. (2005). These methods create blending surface by using rolling-ball method commonly applied for creating blends between two surfaces in a B-Rep model. The blending surface is then meshed and used to replace the part of the mesh to be rounded. The modification is local $\left(\mathrm{a}^{+}\right)$and the quality of the produced mesh is good $\left(\mathrm{c}^{+}\right)$. Configurations involving creases (concave sharp edge) are handled. However, sharp edge terminations and multiple-branches sharp edges cases (Figure 2) refer to as complex configurations not fully supported $\left(\mathrm{e}^{-}\right)$. In the computer graphics and animation domains, several blending approaches have also been proposed but they do not really meet the requirements we have in mechanical engineering (Jin et al., 2006; Whited and Rossignac, 2009) since the mechanical fillet shape could not be ensured $\left(\mathrm{e}^{-}\right)$. Finally, it is important to highlight that none of the above mentioned approaches is able to handle the semantics attached to the mesh elements $\left(\mathrm{f}^{-}, \mathrm{g}^{-}\right)$. Actually, they act on the geometric models without taking care of the associated semantics. The methods modifying locally the mesh will preserve the group definition only in the non-modified area whereas those changing completely the mesh will lose all the group definition. Table I summary all above analysis of all related works according to the declared criteria. Our ambition in the current paper is to design an operator having more "plus" according to each criteria. Moreover, when considering the filleting operation, one can notice that very few methods works on tetrahedral meshes. Thus, the treatment of enriched meshes and the treatment of tetrahedral meshes are two major contributions of this paper.

\begin{tabular}{llllllll}
\hline Criteria & & & & & & & \\
Ref. & a. & b. & c. & d. & e. & f. & g. \\
\hline Premysl (2002) & $\ominus$ & & $\oplus$ & & $\ominus$ & $\ominus$ & $\ominus$ \\
Bremberg and Dhondt (2008) & $\ominus$ & & $\oplus$ & & $\oplus$ & $\ominus$ & $\ominus$ \\
Biermann et al. (2001) & & & & & $\ominus$ & $\ominus$ & $\ominus$ \\
Jung et al. (2004) & $\oplus$ & & $\ominus$ & & & $\ominus$ & $\ominus$ \\
Dakowicz and Gold (2005) & $\oplus$ & & $\ominus$ & & & $\ominus$ & $\ominus$ \\
Turini et al. (2006) & & & $\ominus$ & & $\ominus$ & $\ominus$ & $\ominus$ \\
Chouadria and Veron (2006) & $\oplus$ & & & $\ominus$ & & $\ominus$ & $\ominus$ \\
Chen (2007) & $\ominus$ & & $\ominus$ & $\ominus$ & $\ominus$ & $\ominus$ & $\ominus$ \\
Kim et al. (2004) & $\ominus$ & & & & $\ominus$ & $\ominus$ & $\ominus$ \\
Kim and Yang (2005) & $\oplus$ & & $\ominus$ & & $\ominus$ & $\ominus$ & $\ominus$ \\
Chen et al. (2005) & $\ominus$ & $\oplus$ & & & $\ominus$ & $\ominus$ & $\ominus$ \\
Pavic and Kobbelt (2008) & $\ominus$ & & & & $\ominus$ & $\ominus$ & $\ominus$ \\
Chen and Wang (2011) & $\oplus$ & & & & $\ominus$ & $\ominus$ & $\ominus$ \\
Hui and Lai (2006) & $\oplus$ & & $\oplus$ & & $\ominus$ & $\ominus$ & $\ominus$ \\
Igarashi and Hughes (2003) & $\oplus$ & & $\oplus$ & & $\ominus$ & $\ominus$ & $\ominus$ \\
Lee et al. (2001) & Liv et al. (2005) & & & & $\ominus$ & $\ominus$ & $\ominus$ \\
Jin et al. (2006) & & & & $\ominus$ & $\ominus$ & $\ominus$ \\
Whited and Rossignac (2009) & & & & & & $\ominus$ \\
\end{tabular}

Table I.

Bibliography analysis 


\section{CAD-less mesh operator}

\subsection{General structure of the operator}

The approach proposed in this paper is particularly efficient when prototyping local structural modifications, since it avoids redoing the entire FE mesh semantic enrichment. It is not only very suitable when the original $\mathrm{CAD}$ model is not available, as in the case of maintenance, but also for the preliminary design phases where many alternative solutions often have to be tested. The concept of the CAD-less framework has been proposed in Lou et al. (2010b) and is improved in this paper to better highlight the "generic" character of the CAD-less operators. This framework consists in a general operator structure which relies on sub-operators treating various aspects of the three levels of information (geometry and topology of the mesh, FE mesh group and FE simulation semantics) characterising a FEA mesh model. Gathered together in this generic operator, these sub-operators perform specific changes and/or evaluations of the enriched mesh. Figure 3 illustrates the workflow of the proposed CAD-less framework working on FE mesh models.

\subsection{Details of the operator phases and components}

The general operator workflow consists of three main phases: information processing, geometric processing and semantic processing (Figure 3). The input model gives information of 3 levels: geometry, group and semantics. These information will be used along the three phases (arrows (1), (3) and (5) of Figure 3).

The first phase analyses the initial FE mesh to compute all the information useful for performing the planned mesh modification (Lou et al., 2009, 2010). The information computed includes: boundaries of different categories of the mesh groups contained in the input FE mesh and necessary to link the FE simulation semantics to the mesh elements (see Lou et al, 2009), object shape characteristics, different zones of the mesh required for local mesh deformation process, etc. The considered shape characteristics correspond to particular features such as sharp edges (Lesage et al., 2005) and basic surface shapes like planes, spheres and cylinders (Attene et al., 2006). The identified mesh zones are: the modification area, the unchanged area and the transition zone between them. The transition zone is the nth neighbourhood of the modification area computed by

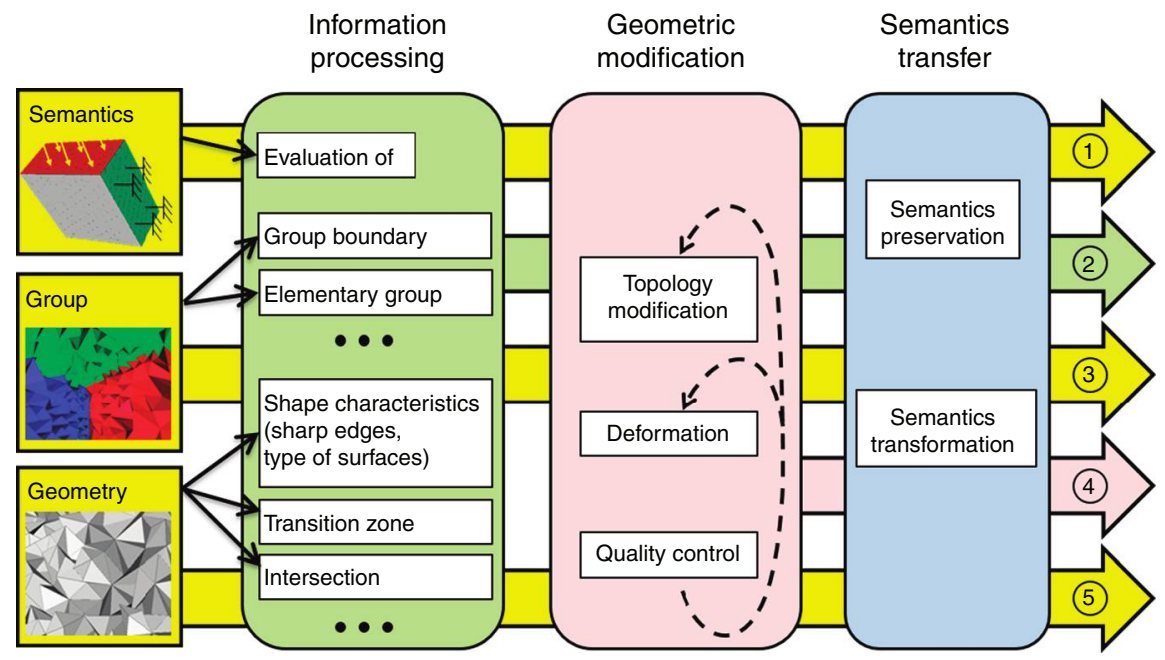

Figure 3.

Three level workflow of the $\mathrm{CAD}$ less framework for manipulating FEA mesh models 
using the mesh connectivity. When the modification is achieved as part addition or removal, the modification area, delimited by the intersection computed between two meshes, must be merged or subtracted (Lou et al., 2010a). All information produced in this phase will be used in the two following phases (arrow (2) of Figure 3).

The second phase performs the actual geometric modifications on the mesh. It takes into account all three levels of information from the initial mesh models (arrows (1), (3) and (5) of Figure 3) and the information resulting from the first processing phase (arrow (2) of Figure 3). At this stage, semantic information evaluated in the first stage can be used for specifying constraints during the mesh modification. The topologic modification consists in adding or removing mesh elements whereas the deformation consists in repositioning the mesh nodes. These modifications are either imposed by a specific mesh operation or launched by quality control. The quality control includes the checking of both the aspect ratio and self-intersection of mesh elements. The modification should preserve as much as possible the group and/or shape characteristics that are computed in the information processing module. To this aim, some prior checks on the mesh can allow a better-quality results. Actually, they can allow the identification of possible problematic cases where mesh refinements would be desirable to obtain compatibility between the mesh element size and the extension of the planned operation. A typical example is the case imposing very restricted rounded shapes on mesh areas with large size elements. This phase generates geometric elements that will be used also later on in the last phase (arrow (4) of Figure 3).

The third phase aims at transferring the FE simulation semantics through the use of preservation and transformation mechanisms. Being given the evaluation of the semantics in the first phase (arrows (2) of Figure 3) as well as the modified geometry (arrow (4) of Figure 3), this phase interacts with the semantics definition. The semantics preservation can correspond to the adequate re-assignment of semantics onto the re-meshed area. Depending on the applied modification and/or the nature of the semantics itself, a simple re-assignment is not possible but a transformation of the semantics is needed, e.g. when the numerical values associated change during the modification process.

\subsection{Instances of the generic operator}

To illustrate the generality of this CAD-less framework, various instances of mesh modification operators have been prototyped taking into account the criteria listed in Section 2. In Lou et al. (2010a), we describe the implemented operator for merging two meshes, while Lou et al. (2010b) presents the drilling and cracking operators for complex 2D and $3 \mathrm{D}$ FE meshes. In this paper, a new instance of the CAD-less operator is presented. It allows the filleting of sharp edges in semantically enriched FE meshes. With this operator the FEA expert can rapidly generate fillets along edges presenting stress concentration, without having to go back to the original CAD model and, consequently, without having to waste time in the re-creation of the corresponding FE model. It is an extension of a previous work (Lou et al. 2012) wherein the filleting of multi-partitioned FE tetrahedral meshes was not possible. Here, our method can fillet FE volume meshes composed of multiple volume partitions that are used to constrain a two-steps deformation process.

\section{Proposed mesh filleting operator}

Roughly speaking, filleting a mesh consists in converting the outer skin neighbouring a set of identified sharp edges into a smooth rounded area. As already stated on the example of the hook (Figure 1), the stress concentrates much more in the sharp areas than in the smooth ones. Therefore, such a filleting operation can be used to improve 
the mechanical behaviour of a structure. Moreover, such an operation can also be used to assess accurately the local stress on an idealized shape. For example, the shape of a welding joint is often simplified and do not always represent the exact shapes. This may lead to inaccurate simulation results.

Therefore, to help engineers directly prototyping an accurate FEA model without going back to the CAD model, having an efficient CAD-less filleting operator can drastically speed up the model preparation steps and also improve the accuracy of the results. As already stated in Section 2, this operator should not only work at the level of the geometric models, but also while exploiting and handling the FE semantic information attached through the use of groups. The proposed sharp edge filleting operator performs the following steps that are detailed in the next subsections:

(1) Identification of the sharp edges to be rounded from user-specified reference edges (Section 4.1, Figure 4(a)).

(2) Identification of the filleting area using the concept of neighbour range to control the extent of the fillet (Section 4.2, Figure 4(b)). The filleting area contains volume elements (e.g. tetrahedra).

(3) Filleting area outer skin deformation (Section 4.5, Figure 4(c)). Here, only the vertices located on the skin of the filleting area are free to move. Therefore, the filleting area inner vertices do not move which may lead to local self-intersections.

(4) Filleting area inner volume relaxation to remove potentially self-intersecting configurations and optimize the aspect ratio of the inner elements (Section 4.6, Figure 4(d)).

From a mechanic point of view, the sharp feature present at an exterior (convex) corner is considered as different from the one at an interior (concave) corner. However, our mesh filleting operator covers the two cases and treats them in a unique way which is not affected by concavity/convexity issues. More precisely, no differences can be seen when treating the two cases, except for the third step where the internal tetrahedrons could stay out of the model hull when it is an exterior corner. Considering the very simple cube (Figure 4(c)), the surface deformation will let the model hull bounding a smaller volume and the internal elements are visible from outside. But this behaviour is controlled and it is discussed in Sections 4.5 and 4.6

\subsection{Sharp edges identification}

To avoid a tedious manual selection of a set of connected edges to be filleted, a dedicated algorithm has been designed. As for the filleting operation on a continuous $\mathrm{CAD}$ model, the idea is to start from a user-specified reference edge and propagate until meeting a stop criterion. Here, the propagation from an edge to its surrounding edges is driven by a user-specified threshold relative to the discrete curvature of the edges. Therefore, the way the curvature of an edge is computed is presented (Section 4.1.1) before developing the overall propagation algorithm (Section 4.1.2).

4.1.1 Discrete curvatures estimation. Among the available approaches for computing discrete curvatures on a polyhedral mesh (Lesage et al., 2005; Gatzke and Grimm, 2006; Mao et al., 2011), the one of Lesage et al. (2005) is interesting since it is invariant to the underlying triangulation and it can be implemented rapidly. In this work, we have extended their approach that was initially designed for estimating discrete curvature at a node. 

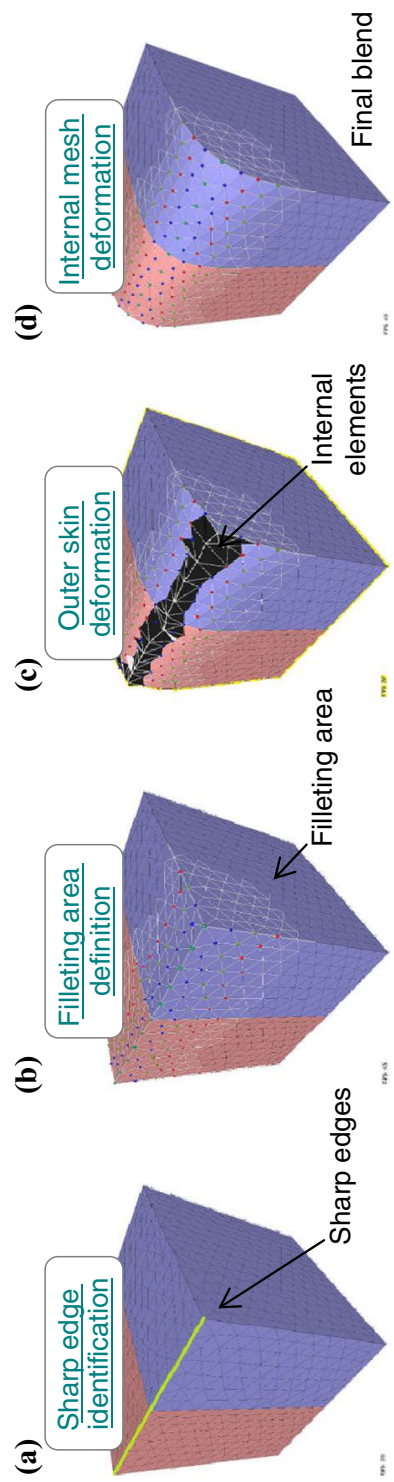

Figure 4.

Example of enriched mesh filleting workflow 
The discrete Gaussian curvature at a node $p$ is given by the following formula wherein $\alpha_{i}$ is the angle of the $i^{\text {th }}$ triangle $\left(f_{i}\right)$ connected to the node $p$ (Figure 5(a)):

$$
I_{K_{p}}=\frac{2 \pi-\sum_{i} \alpha_{i}}{\frac{1}{4} \cdot \sum_{i} \alpha_{j}-\frac{1}{8} \cdot \sum_{i} \alpha_{i}^{2} \cot \left(\alpha_{i}\right)}
$$

Similarly, the discrete mean curvature at the node $\mathrm{p}$ is given by the following formula wherein $\beta_{j}$ is the dihedral angle along the $j$ th edge $\left(e_{j}\right)$ connected to the node $p$ (Figure 5(b)):

$$
I_{H_{p}}=\frac{3}{2} \cdot \frac{\sum_{j} \beta_{j}}{\sum_{i} \alpha_{i}}
$$

Finally, the discrete absolute curvature is given by the following equation:

$$
I_{K_{a b s p}}=4 I_{H_{p}}^{2}-2 I_{K_{p}}
$$

In the proposed approach, the discrete curvature at a node $p$ is estimated by computing its discrete absolute curvature (Equation 3). Then, the discrete curvature of an edge $e$ is computed while taking the smallest discrete absolute curvature of the two end nodes of the considered edge.

4.1.2 Edge propagation algorithm. The following recursive algorithm 1 has been specifically designed to propagate a reference edge and ends up with a list of connected sharp edges.

Since on a discrete mesh, all the edges can potentially be considered as sharp edges, a threshold is used to filter out the smooth edges. The filtering is performed while comparing the discrete absolute curvature of an edge to this user-specified threshold. This parameter is also used to take into account numerical noise due to the mesh discretisation, especially when it has not been generated from a CAD model. Before launching the algorithm, the reference edge is added to the list of sharp edges and the following command is executed:

sharpEdges (reference, threshold, sharp edges)

At the end, the propagation algorithm stops on one of following configurations:

- a so-called end node is shared by only one sharp edge (Figures 6(a) and (b));

(a)

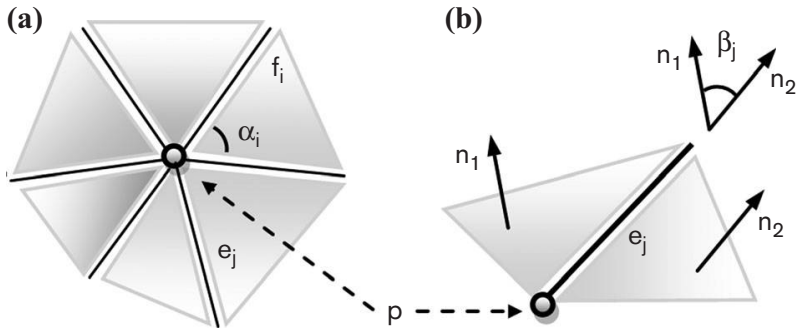

Figure 5.

The angle $\alpha_{i}$ of a triangle $f_{i}$ connected to a reference node $p$ (a), and the dihedral angle $\beta_{j}$ of a reference edge $e_{j}$ (b) 

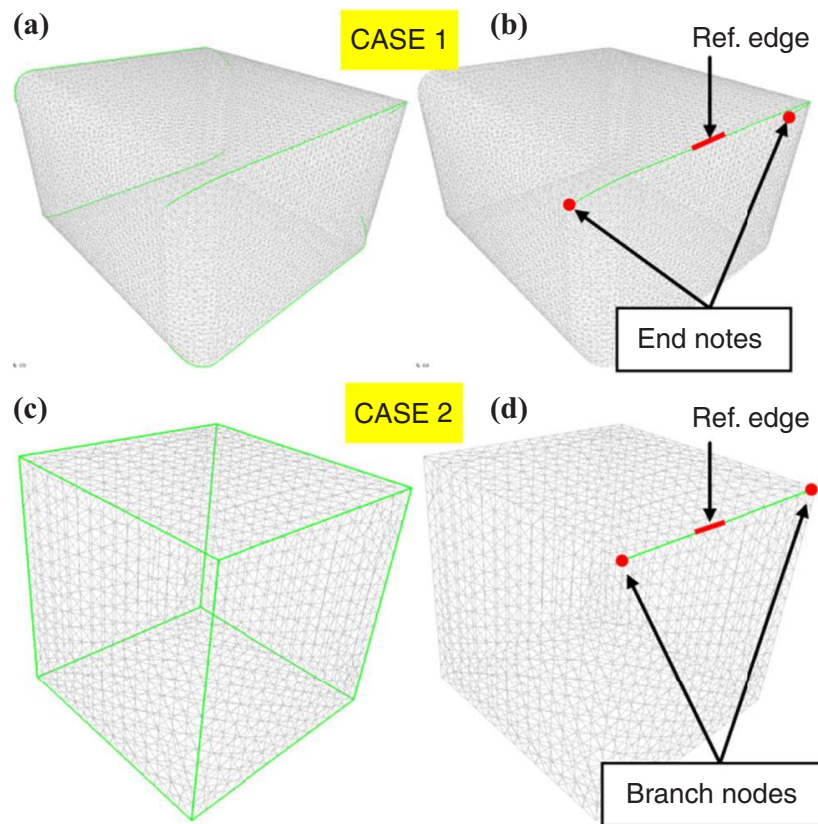

(d) Ref. edge

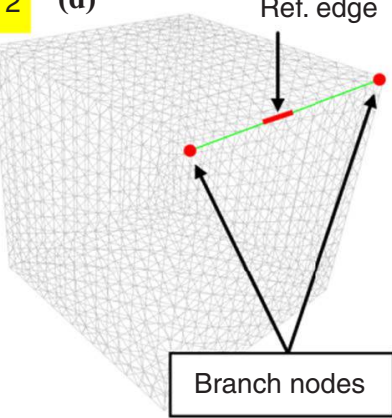

(e)

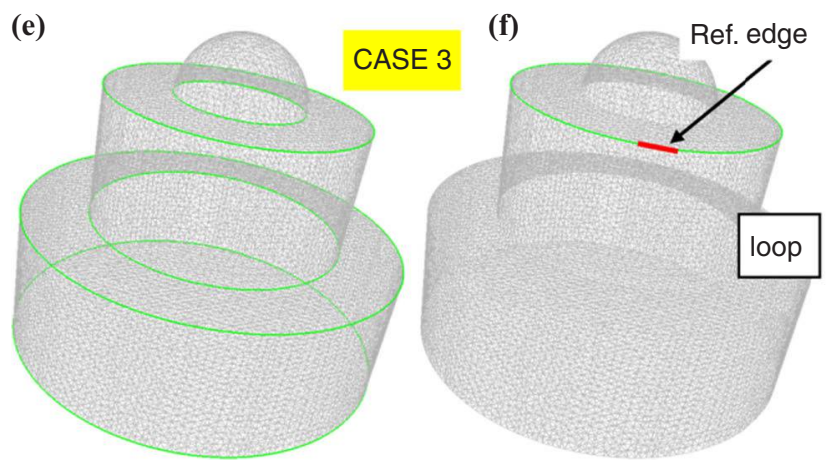

Note: When repeating it several times, all sharp edges can be

Figure 6. selected (a), (c), (e)

The propagation algorithm may end on different configurations (b), (d), (f) 
- a so-called branch node is shared by more than two sharp edges (Figures 6(b) and (c)); and

- a new identified sharp edge was already selected thus forming a loop of sharp edges (Figures 6(e) and 6(f)).

Once the user sees the result, he/she can then still extend the propogation while restarting several times the process with other reference edges:

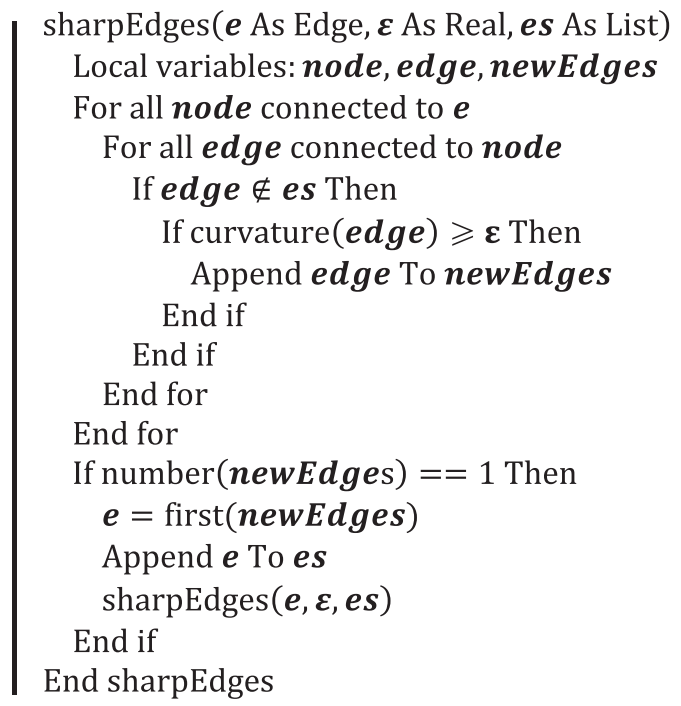

Algorithm 1: sharp edges identification

\subsection{Definition of the filleting area}

Once a connected set of sharp edges has been selected, the filleting area is defined with elements of the neighbourhood. Actually, the nodes surrounding the identified sharp edges can be easily collected while going through the mesh data structure which contains all the information relative to the connections between the mesh geometric entities. These nodes will be used in the deformation process presented in the next subsection. The recursive algorithm 2 illustrates this filleting area definition process. Initially, nodes staying on the identified sharp edges are put into the two lists labelled area and actual. An initially empty list is declared as preced. To control the size of the filleting area, the user gives the number $\boldsymbol{r}$ of neighbour ranges which represents in a discrete manner the radius of the fillet to be generated. The call to the algorithm 2 is performed as follows:

filletArea (area, actual, preced, $r$ )

At the end of the algorithm, all nodes staying in the filleting area are added in the list area. Therefore, in the prototyped filleting operator, the radius of the fillet is not imposed directly, even if it would not be difficult to extend it to take into account 
a specific radius. Note that for a volume mesh the filleting area is specified by the skin and interior nodes around the sharp edges to be filleted:

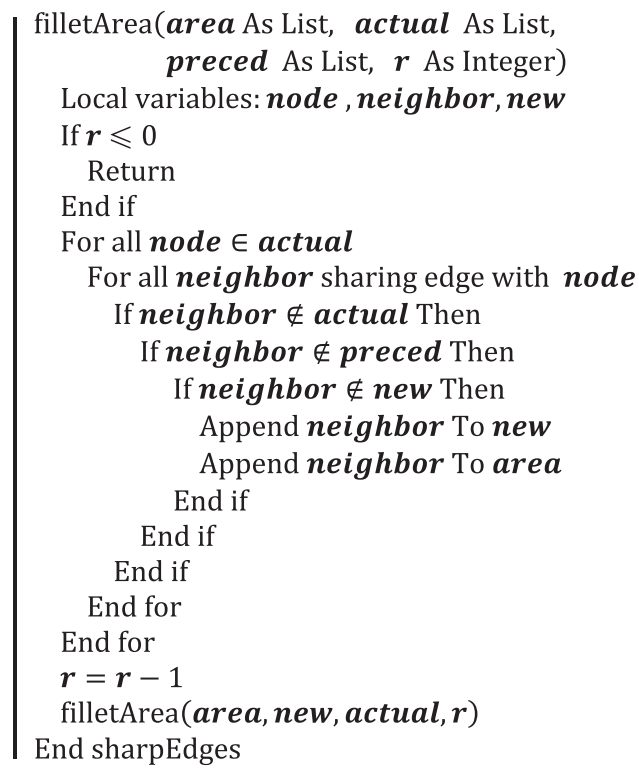

Algorithm 2: Filleting area definition

In Figure 7, starting from a set of selected sharp edges, nodes are identified in areas of different sizes. The nodes in red colour are the ones connected directly to the sharp edges. The algorithm founds the nodes displayed in other colours by using successively $\boldsymbol{r}=2,3$, 4 and 5.

\subsection{Treatment of the groups}

Groups are used to enrich FE meshes with semantic information relative to the simulation process (BCs, material behaviour laws, etc.). To preserve the shape of the groups (criterion $\mathrm{f}$ of Section 2.2), the so-called Virtual Group Boundaries (VGB) have been proposed (Lou et al., 2009). VGB can be extracted in a pre-processing step together with the identification of the type of shape on which the nodes lie (Figure 2). In a 2D mesh (resp. 3D), the VGB is a set of connected edges (resp. faces). Such a VGB bounds the smallest surface (resp. volume) in a 2D (resp. 3D) mesh (Lou et al., 2009) enclosing the group elements. During the mesh modification process, it is important to maintain the shape of these VGB so that semantics can still have the meaning as on the initial configuration. In the prototyped filleting operator, the shape of the VGB is maintain through the use of multiple constraints which take part to the definition of the deformation problem presented in the next subsection. For example, if the VGB of a group is identified as a cylinder, during the deformation, the nodes contained in this VGB will be constrained to stay on the identified cylindrical surface.

Finally, complex simulation models involving configurations where several groups overlap has also been taken into account through the use of Elementary Groups 
(a)

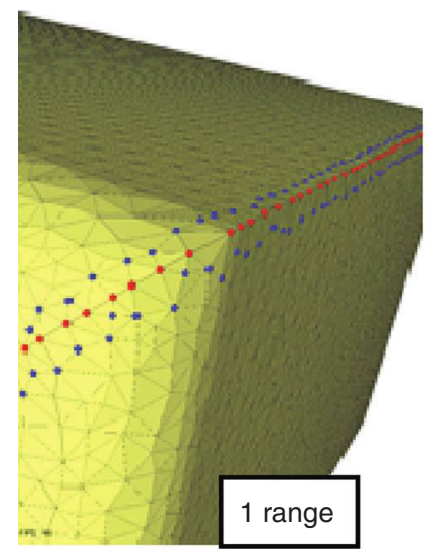

(c)

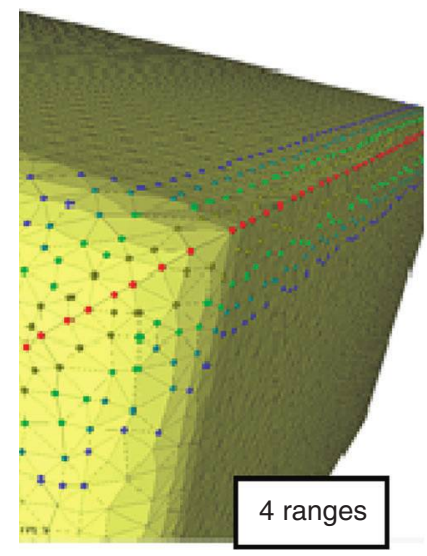

(b)

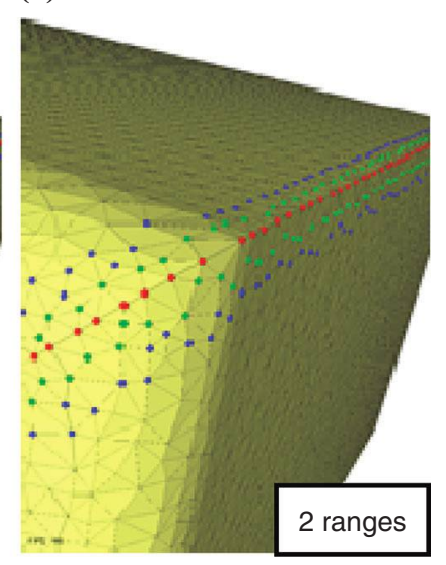

(d)

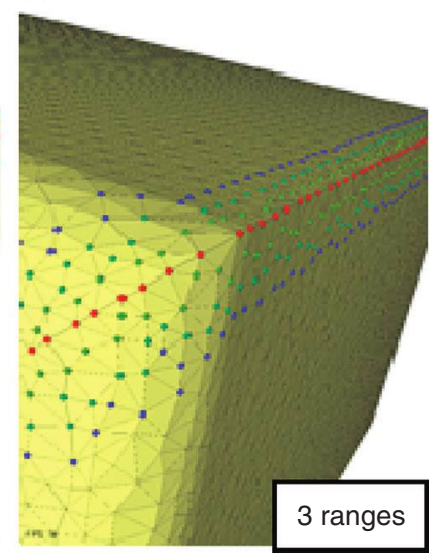

Figure 7.

Different filleting areas

(Lou et al., 2009). Each Elementary Group contains all the elements shared by the same groups.

\subsection{Deformation engine}

In the proposed approach, the filleting of the mesh results from a two-steps deformation process that successively modifies the position of the nodes located on the outer skin of the deformation area (Section 4.5), and then relaxes the inner nodes (Section 4.6). Actually, this decomposition in two distinct steps is due to the adopted deformation engine that is presented in the subsections 4.4.1-4.4.3. Effectively, the coupling of our mechanical model on the overall deformation area would lead to undesired behaviours since the displacements of the inner nodes would affect significantly the shape of the outer skin.

4.4.1 Optimization problem. The nodes displacement results from the resolution of an optimization problem wherein a set of linear and non-linear equality constraints can be imposed to restrict the displacement of identified nodes (notably those nodes either located on the VGB or on the outer skin of the deformation area). Most of the time, the 
system to solve is under-constrained and an additional functional has to be minimized thus defining a complete optimization problem:

$$
\left\{\begin{array}{l}
\mathbf{G}(x)=0 \\
\min \phi(x)
\end{array}\right.
$$

wherein the vector $\boldsymbol{x}$ gathers together the coordinates of the free nodes (i.e. the nodes located in the deformation area), $\boldsymbol{G}(\boldsymbol{x})=0$ is the set of equations constraining some of the nodes, and $\boldsymbol{\phi}(\boldsymbol{x})$ is the function to be minimized.

4.4.2 Objective functions. To produce realistic and smooth fillet, the adopted objective functions are built on top of a linear mechanical model of bar networks coupled to the nodes and edges of the mesh (Pernot et al., 2006). Each edge can be seen as a spring with a null initial length $l_{i}$ and with a stiffness $q_{i}$. Therefore, to maintain the structure in its initial static equilibrium state, external forces have to be applied to the nodes and so that:

$$
\boldsymbol{F}=g(\boldsymbol{x})
$$

wherein $g$ is a linear mapping function linking the external forces $\boldsymbol{F}$ to the coordinates of the free nodes $\boldsymbol{x}$. At the opposite, the position of the free nodes can be obtained from the values of the external forces while using $\mathrm{g}^{-1}$. In our implementation, the positions of the free nodes are considered as the unknown of the optimization problem.

To generate smooth fillet between the deformation area in the blocked area of the mesh, two objective functions are here considered:

(1) the sum of the squared forces applied to the free nodes only:

$$
\phi(x)=\Sigma_{\text {free }} f_{i}^{2}
$$

(2) the sum of the squared forces applied to both the free and blocked nodes:

$$
\boldsymbol{\phi}(\boldsymbol{x})=\Sigma_{\text {free }} \boldsymbol{f}_{i}^{2}+\Sigma_{\text {blocked }} \boldsymbol{f}_{i}^{2}
$$

The example of Figure 8(a) shows a simple structure made of eleven nodes forming a right angle. The four black nodes are blocked and all the other nodes are free to move. As illustrated, to maintain the initial static equilibrium state, three external forces are applied. The minimization of the external forces applied to the free nodes (Equation 6) tends to produce a shape that has a minimal area (actually a minimal length in this $1 \mathrm{D}$ example) as illustrated on Figure 8(b). This minimization is called relaxation since it enables a repositioning of the nodes that minimizes all the forces applied to the structure. If the second objective function (Equation 7) is applied, the sharp corner is rounded with

Figure 8.

Initial mesh (a) and resulting deformation according to the free nodes (b) and all nodes (c) (a)

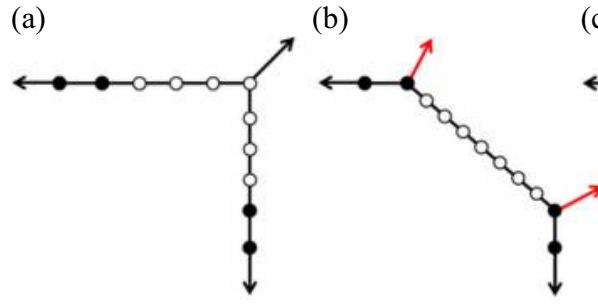

(c)

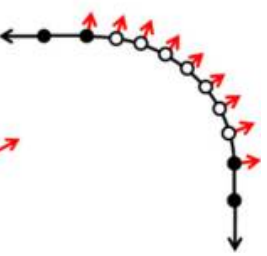


respect to the area defined by the number of free nodes (Figure 8(c)). This minimization tends to minimize the curvature variation between the blocked and free areas.

4.4.3 Constraints specification. To be able to maintain de shape of the VBG, geometric constraints are added to the optimization problem (equation 4). In the actual implementation, free nodes are constrained to stay on planes, spheres and cylinders or on any intersection of these basic primitives:

$$
\begin{aligned}
& G_{p}\left(x_{i}\right)=\left(x_{i}-c\right) \cdot n=0 \\
& \boldsymbol{G}_{\boldsymbol{s}}\left(\boldsymbol{x}_{\boldsymbol{i}}\right)=\left(\boldsymbol{x}_{\boldsymbol{i}}-\boldsymbol{c}\right)^{2}-r^{2}=0 \\
& \boldsymbol{G}_{\boldsymbol{c}}\left(\boldsymbol{x}_{\boldsymbol{i}}\right)=\left[\left(\boldsymbol{x}_{\boldsymbol{i}}-\boldsymbol{c}\right)^{\hat{n} \boldsymbol{n}}\right]^{2}-r^{2}=0
\end{aligned}
$$

wherein $\boldsymbol{x}_{\boldsymbol{i}}$ is the point that is constrained. The planar constraint restricts the displacement of $\boldsymbol{x}_{\boldsymbol{i}}$ to the plane defined by a point $\boldsymbol{c}$ and a normal $\boldsymbol{n}$. The spherical constraint imposes $\boldsymbol{x}_{\boldsymbol{i}}$ to lie on a sphere of radius $\boldsymbol{r}$ centred in $\boldsymbol{c}$. Finally, the cylindrical constraint restricts the displacement of $\boldsymbol{x}_{\boldsymbol{i}}$ to a cylinder defined by its radius $r$ and axis $\boldsymbol{n}$ going through a point $\boldsymbol{c}$.

\subsection{Surface mesh deformation}

As introduced in Section 4.4, the filleting operator works in two successive deformation steps: the outer skin deformation and the inner volume mesh relaxation. Both steps are applied on the identified deformation area gathering together mesh elements in the surrounding of identified sharp edges.

The first step aims at repositioning the nodes located on the outer skin of the deformation area to smooth the normal evolution between the initial and deformed areas. Such a smoothing is obtained while using the objective function of Equation 7. This is illustrated on the example of Figure 9 which shows a section of a filleting area of a tetrahedral mesh. The two cases of convex sharp corner (upper) and concave sharp

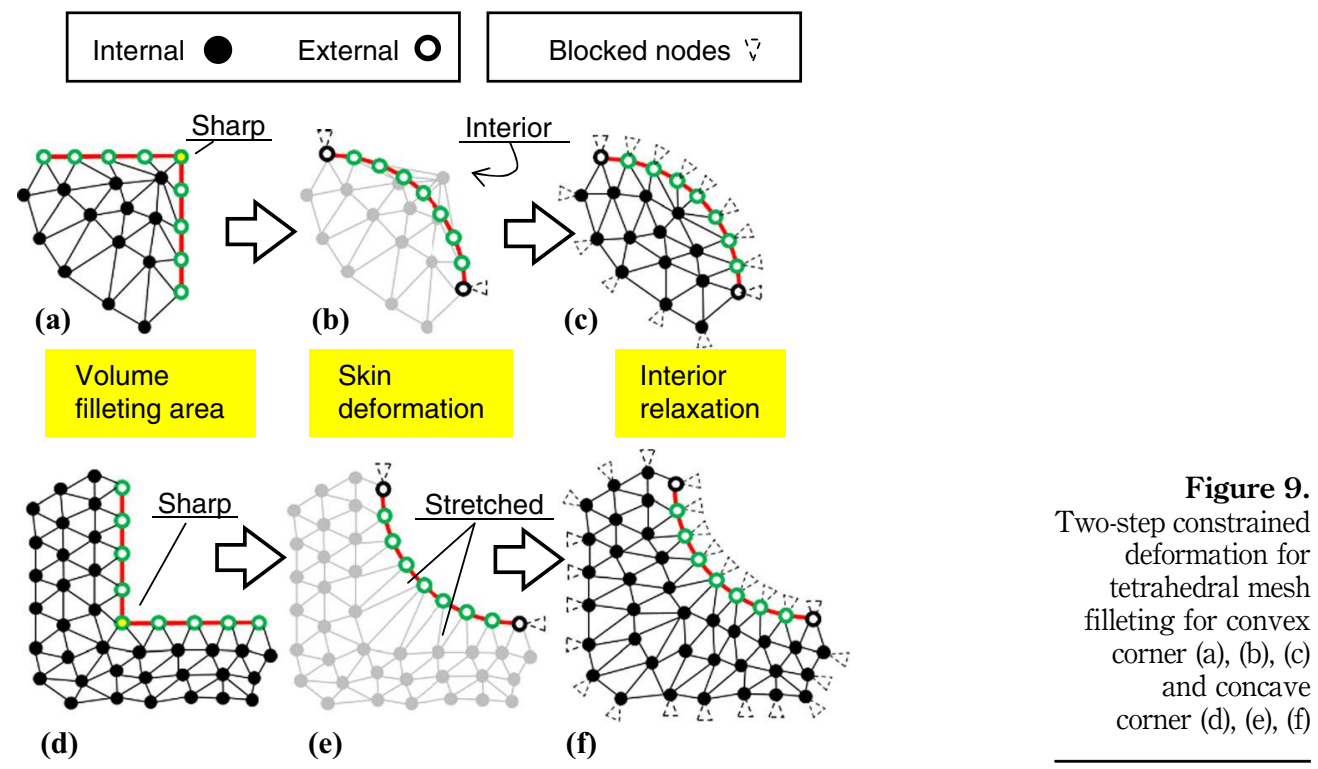


corner (lower) are both illustrated in the Figure 9. Figure 9(a) and (d) correspond to the initial configuration before deformation. The black edges are internal and the red edges are skin elements. As illustrated on Figure 9(b) and (e), solely the nodes located on the skin of the deformation area are free to move. As highlighted on Figure 3 (c), solely the outer skin of the deformation area is smoothed thus producing several intersections between the skin and the inner elements (in dark on Figure 9) which have not yet been relaxed. In the Figure 9 (b) the same phenomena can be seen: some sharp edge neighbour internal elements are coming out from the skin. In an opposite way, when the sharp corner is concave at beginning (Figure 9 (d)), the internal elements close to the sharp edges are stretched after the model skin deformation.

During the deformation process, some of the free nodes are also constrained to stay on certain surfaces in order to preserve the shape of the initial model and the VGB. As stated in Section 3.2, before modifying the FE meshes our CAD-less framework will compute the primitive surfaces for the initial models as well as for the VGB (Section 4.3). The constraints are defined on the free nodes using the equations developed in Section 4.4.3. Thus the shapes of the model and of the groups can be preserved.

Figure 10 compares the results of deformation with and without constraints for the example already illustrated in Figure 3. During the skin rounding deformation, the group boundary consists of a set of yellow edges that separate the two groups. The VGB shape is broken (Figure 10 (a)) when the free nodes have moved without constraints whereas the constrained deformation allows to preserve the shape of VGB (Figure 10 (b)).

Beside the shape of VGB the shape of the initial model is also concerned in this example. All the nodes on the two lateral sides should stay on the lateral plans in order to keep the initial shape. If any constraints is defined on the free nodes (Figure 10 (c)) the initial shape is broken and the lateral sharp curves (yellow edges) disappeared which should not be filleted. Figure 10 (d) shows the deformation under constraints where the lateral surface bounded by the yellow edges are kept planar.

\subsection{Volume mesh relaxation}

In the second deformation step, the nodes of the deformation area that have been blocked during the first step are now free to move to adapt their positions inside the volume mesh, whereas the nodes of the outer skin are now blocked. Here, we use the objective function of Equation 6 to relax the positions of the nodes.

Coming back to the example of Figure 9, and after the outer skin deformation step (Figure 9 (b) and (e)), the volume mesh relaxation step is performed so that the position of the deformation area inner nodes are relaxed (Figure 9(c) and (f)). Here, no constraints are applied, and the optimization problem consists in the minimization of a quadratic function. On the example of Figure 3, a similar configuration is obtained on the inner nodes of a volume deformation area. On this example, the inner free nodes are relaxed, and some of them are also constrained to stay on the plane defined by the VGB between the two partitions (Figure 4(d)). The Figure 10(f) displays solely the red volume group and the faces bounded by the yellow edges composing the constrained VGB. If no constraint is defined on the free nodes, the VGB faces close to the filleting area have lost their initial shape (Figure 10(e)).

\section{Results on academic and industrial examples}

In this section we report the results of the filleting operator applied to both academic and industrial semantically enriched parts. 

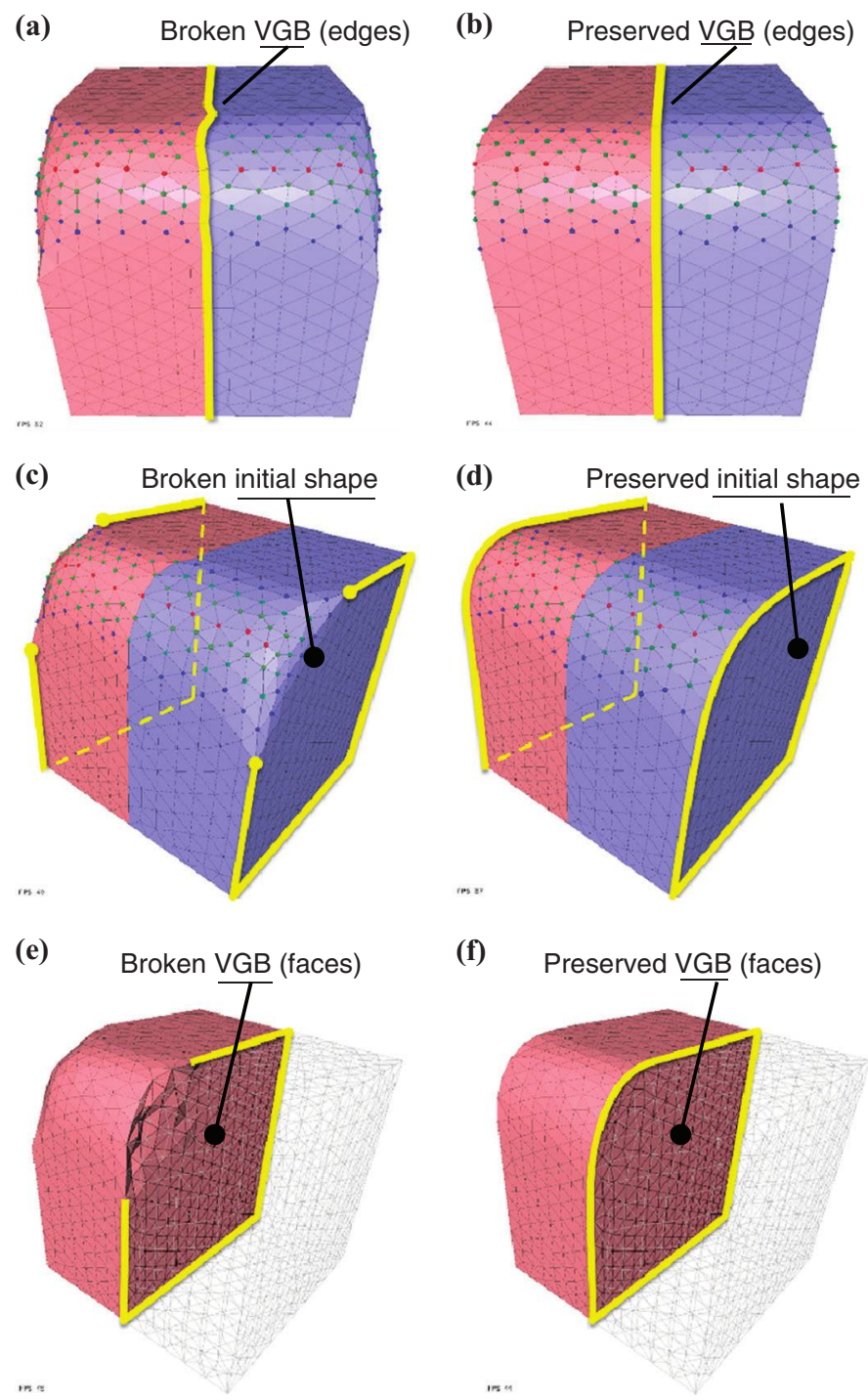

Figure 10.

Filleting mesh deformation without (a), (c), (e) and under (b), (d), (f) constraints of model shape and virtual group boundary (VGB)

The first presented CAD-less filleting experimentation is applied on a tetrahedral mesh, on which two groups (G1 and G2) of tetrahedrons are defined (Figure 11(a)). The objective is to fillet the sharp edges between the two cylinders (Figure 11(b) and (d)). The filleting area is defined by two neighbourhood ranges (§ 4.2). Figure 11(c) and (e) show the result of the deformation where the sharp edges disappear. As previously described, the deformation process consists of two stages. The first stage deforms the boundary surface of the filleting area into a round shape $(\S 4.5)$. Then the second deformation relaxes the tetrahedrons within the filleting area (§ 4.6). In Figure 11(b) and (c) only the tetrahedrons belonging to group $\mathrm{G} 2$ are coloured, while those belonging to G1 are depicted in wire frame mode to more clearly show the quality and the actual 

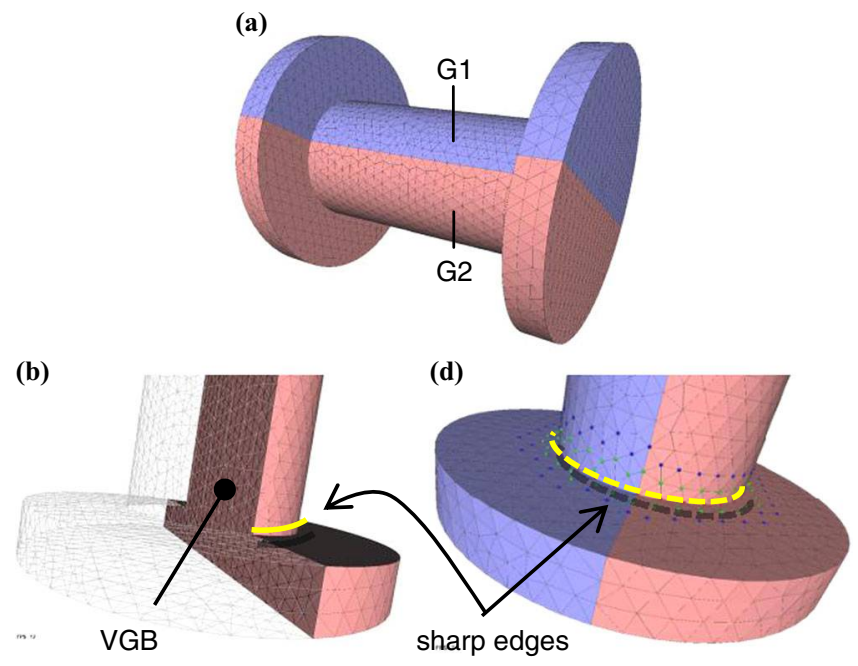

Figure 11.

CAD-less filleting on a partitioned tetrahedral mesh (c)

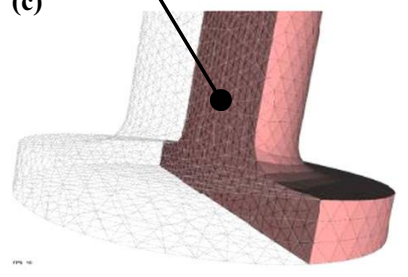

(e)

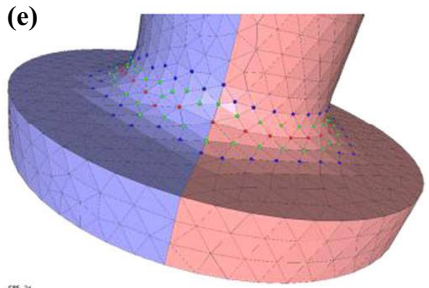

group association in the resulting mesh. As depicted, the triangles defining the group boundary of G1 and G2 (cf. VGB in Section 4.3) are inside the model and still maintain their initial shape (e.g. a plane in the present case).

In the second example, the presented CAD-less filleting experimentation is applied on a tetrahedral mesh on which three groups (G1, G2 and G3) of tetrahedron are defined (Figure 12(a)). The sharp edge to round is between the group G2 and G3 (Figure 12(c)). Figure 12(b) shows the partial view of the initial model interior. In this example 5 neighbourhood ranges are defined as filleting area (Figure 12(c)). The nodes selected in the filleting area are displayed as bold green and blue points. Figure 12(e) shows the result of the deformation. The first stage deforms the boundary surface of the filleting area into a round shape (Figure 12(d)) and any node inside is moved at this stage. Then, the second deformation repositions the internal nodes to relax the stretched tetrahedrons due to surface deformation (Figure 12(f)). In this example the virtual group boundaries (VGB) that are inside the model and separate the two groups G2 and G3 maintain their initial shape (as shown in Figure 12(f)). At the same time, those external VGB nodes belonging to the filleted area have been constrained to simultaneously be on the round shape defined by the fillet and on the planar surface defined the original VGB of the G2 and G3.

The third example (Figure 13(a)) consists in filleting sharp edges inside a caisson model. This model contains four tetrahedral groups that are displayed in four different colours. Figures 13(b), (c) and (d) give top views from the model. The sharp edges are displayed in Figure 13(c) and correspond to the connection with the bottom face of caisson interior. On the partial view on the model where only the red group is displayed 


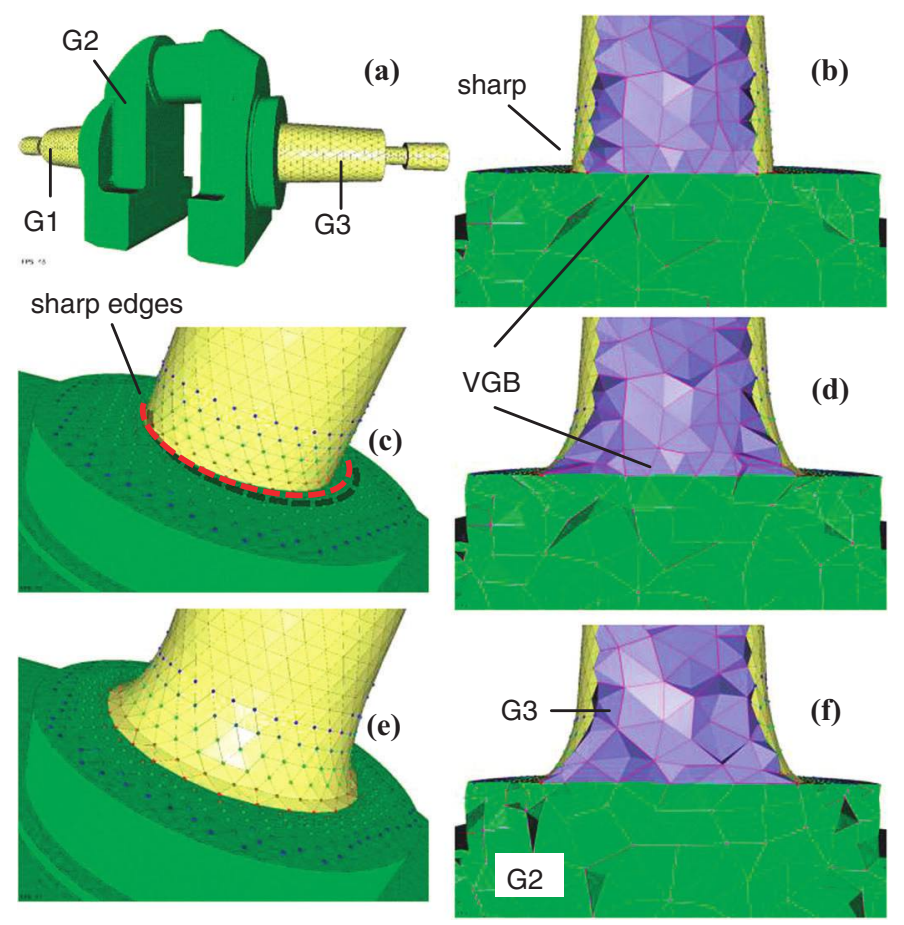

Figure 12. CAD-less filleting on a tetrahedral mesh

(Figure 13(e)), the sharp edges are coloured in yellow. The deformed model is shown entirely (Figure 13(d)) and partially (Figure 13(f)). Also on this example we can see that the shape of the VGB is also preserved.

Finally, Table II summarizes the information relative to each of the examples shown in this paper: the numbers of mesh elements and the ranges defining the filleting zone $(\S 4.2)$. The initial and final qualities in terms of aspect ratios for each example before and after operation are also detailed, showing an improvement. The aspect ratio of a tetrahedron is computed according to the method presented in Bern and Plassmann (2000). One can notice that the initial quality of the various configurations is well preserved which is important for the forthcoming simulation step.

\section{Conclusion}

In this paper, we set up a new framework to enable direct modifications of volume meshes enriched with semantic information associated to multiple partitions. Such a CAD-less approach allows a much faster generation and assessment of alternative solutions than the classical process. Effectively, since the modifications are directly performed onto the enriched FE meshes, we avoid time-consuming loops between the $\mathrm{CAD}$ models modification and $\mathrm{FE}$ models preparation. If at the end CAD models are required, it is still possible to generate one, or update an existing one, but this will be done just once. With respect to the Isogeometric Analysis approach, our solution allows engineers to still use the FEA methods they trust and normally use while avoiding the transfer from $\mathrm{CAD}$ to $\mathrm{FE}$ models.

To illustrate the capability of the framework, the so-called mesh filleting operator has been introduced. It rounds the surroundings of a set of user-specified sharp edges. 
Figure 13.

CAD-less filleting on a tetrahedral mesh (a)

(b)
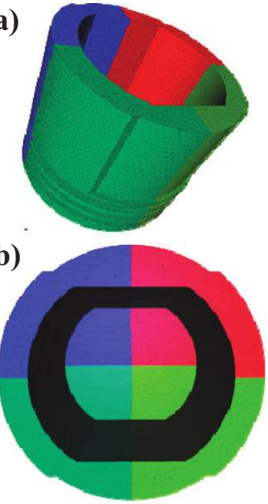

(c)

(d)

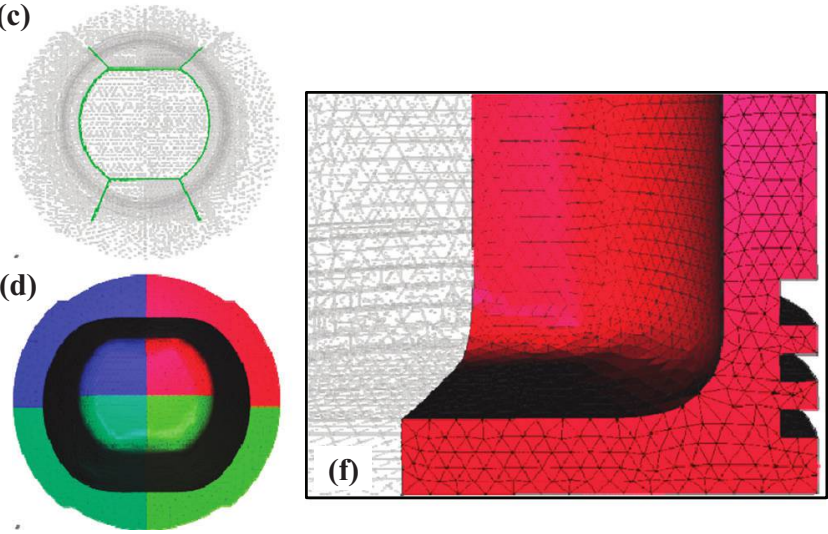

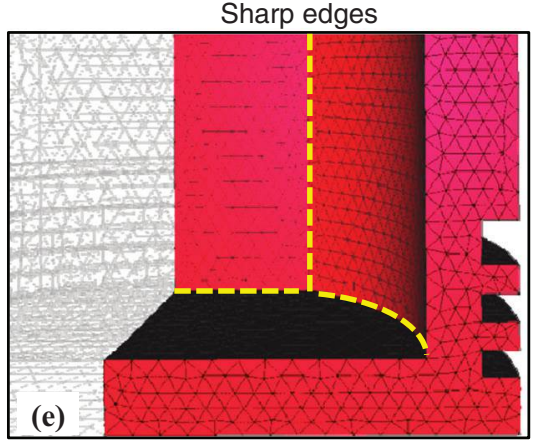

(e)

\begin{tabular}{lcccc}
\hline $\begin{array}{l}\text { Meshes } \\
\text { Criteria }\end{array}$ & $\begin{array}{c}\text { Cube } \\
\text { (Figure 4) }\end{array}$ & $\begin{array}{c}\text { Cylinder } \\
\text { (Figure 11) }\end{array}$ & $\begin{array}{c}\text { Meca } \\
\text { (Figure 12) }\end{array}$ & $\begin{array}{c}\text { Caisson } \\
\text { (Figure 13) }\end{array}$ \\
\hline Nb. nodes & 2,244 & 4,468 & 21,805 & 16,733 \\
Nb. tetras & 9,598 & 18,938 & 92,985 & 66,777 \\
Range & 3 & 2 & 5 & 3 \\
Q & 0.698 & 0.675 & 0.705 & 0.657 \\
Q $_{\text {final }}$ & 0.690 & 0.669 & 0.700 & 0.649
\end{tabular}

The final shape results from a two-step deformation process which first works on the skin of the mesh before relaxing inner nodes in an identified deformation area. During the smoothing process, some nodes might have to fulfil additional constraints depending on whether they are located on a VGB or not. Therefore, this operator not only works at the level of the mesh elements, but also takes into account semantic information attached to multiple partitions of the mesh. This is a real breakthrough in the way FE meshes can be manipulated directly with respect to the existing approaches, i.e. guaranteeing the mesh geometric quality and preserving the $\mathrm{FE}$ knowledge during the mesh modification steps.

However, several improvements are foreseen. At the geometric level, the deformation area, i.e. the part of the mesh that has to be rounded, will be computed 
directly from a user-specified filleting radius. Actually, along the sharp edges, the number of ranges used to define the deformation area should vary according to the density of the surrounding mesh elements. To better approximate the user-specified filleting radius, whatever the mesh density is, the use of mesh enrichment could be exploited. Moreover, new developments for overcoming problems due to noisy meshes in the sharp edges computation (i.e. propagation in the surrounding of a user-specified reference sharp edge) will be undertaken.

At the semantic level, future works concern the improvement of the treatment and transfer of the semantic information through a set of rules such as inheritance, propagation, etc. Actually, the actual developed filleting operator does not yet work on the $\mathrm{FE}$ semantics attached to the groups but rather on the type of shape associated to VGB which is already an improvement in the way enriched FE meshes are manipulated.

Finally, such a modelling approach could also be extended to other operators or to other simulations such as topological optimization.

\section{References}

Attene, M., Falcidieno, B. and Spagnuolo, M. (2006), "Hierarchical mesh segmentation based on fitting primitives", L. Visual Computer, Vol. 22 No. 3, pp. 181-193.

Bern, M. and Plassmann, P. (2000), "Mesh generation”, in Sack, I.-R. and Urruita. I. (Eds), Handbook of Computational Geometry, Elsevier Science Publishers B.V., North-Holland, Amsterdam, pp. 291-332.

Biermann, H., Kristjansson, D. and Zorin, D. (2001), "Approximate boolean operations on free-form solids", Proc. of the 28th Annual Conference on Computer graphics and Interactive Techniques, SIGGRAPH '01, pp. 185-194.

Bremberg, D. and Dhondt, G. (2008), "Automatic crack-insertion for arbitrary crack growth", Engineering Fracture Mechanics, Vol. 75 Nos 3/4, pp. 404-416.

Chen, Y. (2007), "Robust and accurate boolean operations on polygonal models", Proc. of ASME Design Engineering Technical Conferences, Volume 2: 27th Computers and Information in Engineering Conference, Las Vegas, Nevada, 4-7 September.

Chen, Y. and Wang, C.C.L. (2011), "Uniform offsetting of polygonal model based on layered depth-normal images", Int. I. CAD, Vol. 43 No. 1, pp. 31-46.

Chen, Y., Wang, H., Rosen, D.-W. and Rossignac, J. (2005), "Filleting and rounding using a point-based method", Proc. of DETC'05, ASME Design Engineering Technical Conferences and Computers and Information in Engineering Conference, Long Beach, CA, 24-28 September, pp. 1-10.

Chouadria, R. and Veron, P. (2006), "Identifying and re-meshing contact interfaces in a polyhedral assembly for digital mock-up", L. of Engineering with Combuter, Vol. 22 No. 1, pp. 47-58.

Ciarlet, P.G. (1978), The Finite Element Method for Elliptic Problems, North-Holland, Amsterdam, New York, Oxford.

Cottrell, J.A., Hughes, T.J.R. and Bazilevs, Y. (2009), Isogeometric Analvsis: Towards Integration of CAD and FEM, John Wiley \& Sons, Chichester.

Dakowicz, M. and Gold, C. (2005), "Interactive tin modification with a cutting tool", Proc. of 4th ISPRS Workshop on Dynamic and Multi-dimensional GIS, Pontypridd, Wales, pp. 5-9.

Gatzke, T. and Grimm, C. (2006), "Estimating curvature on triangular meshes", Int. J. IJSM, Vol. 12 No. 1, pp. 1-29.

Hui, K.C. and Lai, Y.H. (2006), "Smooth blending of subdivision surfaces", Int. I. CAD, Vol. 38 No. 7, pp. 786-799.

Igarashi, T. and Hughes, J.F. (2003), "Smooth meshes for sketch-based freeform modeling", Proc. ACM Svmposium on Interactive 3D Graphics, Monterey, CA, pp. 139-142. 
Jin, X., Lin, J., Wang, C.C.L. and Feng, J. (2006), "Hanqiu sun: mesh fusion using functional blending on topologically incompatible sections", Visual Computer, Vol. 22 No. 4, pp. 266-275.

Jung, W., Shin, H. and Choi, B.K. (2004), "Self-intersection removal in triangular mesh offsetting", Computer-Aided Design and Applications, Vol. 1 Nos 1/4, pp. 477-484.

Kim, S.-J. and Yang, M.-Y. (2005), "Triangular mesh offset for generalized cutter”, Int. J. CAD, Vol. 37 No. 10, pp. 999-1014.

Kim, S.J., Lee, D.Y. and Yang, M.Y. (2004), "Offset triangular mesh using the multiple normal vectors of a vertex", Computer-Aided Design and Applications, Vol. 1 Nos 1/4, pp. 285-292.

Lee, S.H., Lee, W.K. and Lee, K.-S. (2001), "Rounding operations on shell meshes for efficient analysis of stamping tools for automotive body panels", Proc. of the 11th International Pacific Conference on Automotive Engineering (IPC-11), 6-9 November.

Lesage, D., Léon, J.-C. and Véron, P. (2005), “Discrete curvature approximations and segmentation of polyhedral surface”, Int. J. IJSM, Vol. 11 No. 2, pp. 217-252.

Liu, Y., Zhang, H., Yong, J., Yu, P. and Sun, J. (2005), "Mesh blending", The Visual Computer, Vol. 21 No. 11, pp. 915-927.

Loop, C. (1987), "Smooth subdivision surfaces based on triangles", M.S. Mathematics thesis, University of Utah, Salt Lake City, UT.

Lou, R., Mikchevitch, A., Pernot, J.-P. and Véron, P. (2010a), "Merging enriched finite element triangle meshes for fast prototyping of alternate solutions in the context of industrial maintenance", Int. J. CAD, Vol. 42 No. 8, pp. 670-681.

Lou, R., Pernot, J.-P., Giannini, F., Véron, P. and Falcidieno, B. (2012), "Sharp edge filleting of enriched FE meshes", Proc. of TMCE 2012, Karlsruhe.

Lou, R., Giannini, F., Pernot, J.-P., Mikchevitch, A., Véron, P., Falcidieno, B. and Marc, R. (2009), "Towards CAD-less finite element analysis using group boundaries for enriched meshes manipulation", Proc. of ASME 2009 IDETC \& CIE, Vol. 2, San Diego, CA, pp. 29-38.

Lou, R., Giannini, F., Pernot, J.-P., Mikchevitch, A., Véron, P., Falcidieno, B. and Marc, R. (2010b), "Direct modification of semantically-enriched finite element meshes", Int. J. IJSM, Vol. 16 Nos 1/2, pp. 81-108.

Mao, Z., Cao, G., Ma, Y. and Kunwoo, L. (2011), "Curvature estimation for meshes based on vertex normal triangles”, J. Computer-Aided Design, Vol. 43 No. 12, pp. 1561-1566.

Pavic, D. and Kobbelt, L. (2008), "High-resolution volumetric computation of offset surfaces with feature preservation”, Computer Graphics Forum, Vol. 27 No. 2, pp. 165-174.

Pernot, J.-P., Moraru, G. and Véron, P. (2006), "Filling holes in meshes using a mechanical model to simulate the curvature variation minimization", Computers \& Graphics, Vol. 30 No. 6, pp. 892-902.

Premysl, K. (2002), "Complex human tissues fem models prepared by boolean operations", Biomechanics of Man, Faculty of Physical Education and Sport Charles University in Prague, pp. 24-26.

Turini, G., Ganovelli, F. and Montani, C. (2006), "Simulating drilling on tetrahedral meshes", Proc. of Eurographics Conference, pp. 127-131.

Whited, B. and Rossignac, J. (2009), "Relative blending”, Computer-Aided Design, Vol. 41 No. 6, pp. 456-462.

\section{Corresponding author}

Assistant Professor Ruding Lou can be contacted at: ruding.lou@ensam.eu 